\title{
Radiopacidad de Resinas Acrílicas y Bisacrílicas Usadas como Restauraciones Provisionales
}

\author{
Radiopacity of Acrylic and Bisacrylic Resins Used as Provisional Restorations
}

Vicente Vega1; Sylvia Osorio ${ }^{1,2}$; Juan Estay¹,2; José Farfán¹; Constanza Quintana1 \& Camila Corral ${ }^{1}$

VEGA, V.; OSORIO, S.; ESTAY, J.; FARFÁN, J.; QUINTANA, C. \& CORRAL, C. Radiopacidad de resinas acrílicas y bisacrílicas usadas como restauraciones provisionales. Int. J. Odontostomat., 15(1):119-124, 2021.

RESUMEN: Una adecuada radiopacidad de los materiales para restauraciones provisorias permite su evaluación en el examen radiográfico. Sin embargo, existe gran desconocimiento sobre el cumplimiento de los requerimientos establecidos por la norma ISO 4049. El objetivo del siguiente estudio fue determinar la radiopacidad de los materiales para provisionalización presentes en el mercado nacional y corroborar si estos cumplen con el valor mínimo requerido por la normativa. Se realizó un estudio experimental in vitro, en el cual se prepararon muestras en forma de discos de 10 mm de diámetro y $1 \mathrm{~mm}$ de espesor de resinas acrílicas: Acrílico Autocurable (Marché®), Duralay (Reliance), Caulkâ (Dentsply), $\operatorname{ALIKE}^{\text {TM }}$ (GC) y resinas bisacrílicas: Protemp ${ }^{\text {TM }} 4$ (3M, 2008), LuxatempStar (DMG), Luxacrown (DMG), Structur Premium (VOCO), ExperTemp ${ }^{\mathrm{TM}}$ (Ultradent) y Acrytemp (Zhermack). Las muestras fueron radiografiadas junto a una cuña de aluminio escalonada. Las imágenes fueron analizadas para determinar el valor en escala de grises y su respectivo valor en mm de aluminio. Los datos fueron analizados para verificar si cumplen con la normativa ISO. Se encontraron diferencias estadísticamente significativas. La radiopacidad equivalente de las resinas acrílicas en mm de Al en orden descendente fue de $0,85 \pm 0,08$ para ALIKE ${ }^{\text {TM }}, 0,72 \pm 0,32$ para acrílico Marché $\circledast, 0,65 \pm 0,25$ para Caulkâ y 0,56 $\pm 0,05$ para Duralay, siendo todos menores a lo requerido por la norma. La radiopacidad equivalente de las resinas bisacrílicas fue de 1,75 $\pm 0,2$ para Acrytemp, 1,51 \pm 0,27 para Luxacrown, 1,47 \pm 0,14 para Structur Premium, 1,43 \pm 0,23 para LuxatempStar, 1,02 $\pm 0,28$ para Protemp ${ }^{\mathrm{TM}} 4$ y $1 \pm 0,1$ para ExperTemp ${ }^{\mathrm{TM}}$, los cuales son mayores a lo exigido por la norma. En conclusión, las resinas bisacrílicasevaluadas en este estudio cumplen con el requisito de radiopacidad exigido, a diferencia de las acrílicas que no lo cumplen.

PALABRAS CLAVE: radiopacidad, materiales dentales, resinas acrílicas.

\section{INTRODUCCIÓN}

En general, todos los materiales dentales deberían poseer una radiopacidad suficiente para poder distinguirlos del diente y estructuras adyacentes en una radiografía (Turgut et al, 2003). Sin embargo, con respecto a los materiales para confección de restauraciones provisionales, la mayoría de las investigaciones se enfoca solo en analizar sus propiedades estéticas y mecánicas, por tanto, no existe mucha evidencia respecto a su radiopacidad. Esta propiedad es relevante ya que permite en restauraciones provisorias evaluar características de difícil análisis clínico, como son el ajuste marginal y los contornos interproximales (Gómez Mira \& Ardila Medina, 2009). Además, es tam- bién relevante en casos de accidentes por aspiración o impactación, poder facilitar su ubicación con radiografías (Mattie et al., 1994; Romanos, 2004).

La International Organization for Standarization (ISO), establece la norma 4049 para regular y establecer las propiedades que deben poseer los materiales restauradores basados en polímeros, incluyendo los protocolos necesarios para estudiar cada una de ellas. Plantea que los materiales restauradores (sin distinción de provisorios o definitivos) deben tener una radiopacidad igual o mayor a la equivalente a $1 \mathrm{~mm}$ de aluminio. Además, si el fabricante determina que el

\footnotetext{
${ }^{1}$ Departamento de Odontología Restauradora, Facultad de Odontología, Universidad de Chile, Chile.

${ }^{2}$ Departamento de Patología y Medicina Oral, Facultad de Odontología, Universidad de Chile, Chile.
}

Received: 2020-06-26 Accepted: 2020-09-23 
material posee una cierta radiopacidad, la radiopacidad no debe ser menor a $0,5 \mathrm{~mm}$ de aluminio de lo publicitado (International Organization for Standardization, 2009). Diferentes estudios que analizan la radiopacidad de los materiales dentales concluyen que es necesaria una mayor regulación respecto al criterio que utiliza cada fabricante para definir la radiopacidad, sugiriendo que sea según la indicación del material y los tejidos adyacentes (Pekkan, 2016).

Actualmente, no todos los materiales usados para provisionalización se encuentran debidamente regulados en relación a su radiopacidad. Por este motivo es que el presente estudio reporta la radiopacidad de distintos materiales para confeccionar provisionales que se distribuyen en el mercado nacional, utilizando un protocolo estandarizado (Vega et al., 2017) que se ajusta a los requerimientos establecidos por la normativa ISO. Con la finalidad de ser una referencia tanto para el clínico a la hora de elegir un material como para el fabricante a la hora de estandarizar y publicitar la radiopacidad de sus materiales.

\section{MATERIAL Y MÉTODO}

Esta investigación consistió en un estudio experimental in vitro, donde se determinó y comparó la radiopacidad de resinas acrílicas y bisacrílicas paraconfección de restauraciones provisionales. Los materiales evaluados se muestran en Figura 1.
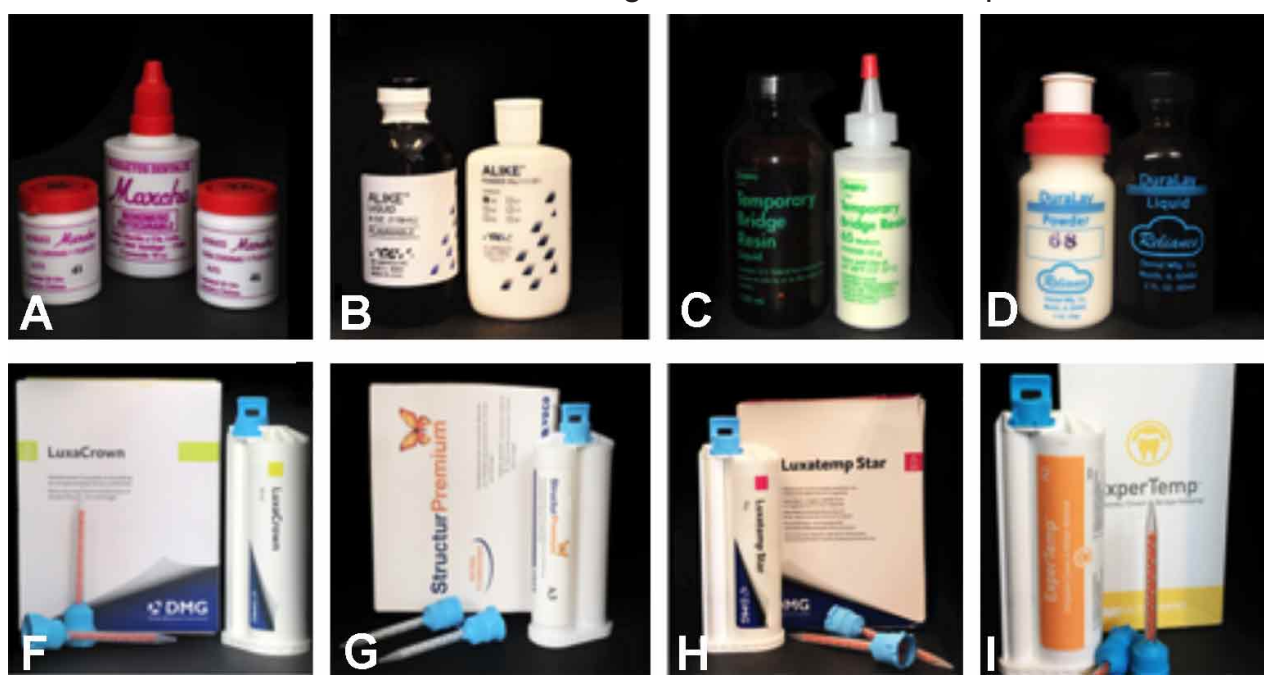

Evaluación de la radiopacidad. El test de radiopacidad y la preparación de los materiales fueron llevados a cabo acorde a las indicaciones de las normas ISO 4049. Los materiales fueron mezclados siguiendo las instrucciones de los fabricantes, y posicionados en moldes que presentaban agujeros de $1 \mathrm{~mm}$ de grosor y $10 \mathrm{~mm}$ de diámetro. El grosor se corroboró mediante pie de metro digital (Pie de metro E5001002, Veto, Chile) y se descartaron las muestras que presentaron burbujas o defectos.

Cinco muestras de cada material fueron posicionadas en una película digital de fósforo fotoestimulable (PSP, $48 \times 54 \mathrm{~mm}$, FireCR Dental, 3DISC Corp., Daejeon, Korea) adyacentes a una cuña de aluminio escalonada de 1 a $10 \mathrm{~mm}$, con pureza mayor a 99 \% de Aluminio (Odeme, Santa Catarina, Brasil). La toma radiográfica se estandarizó utilizando un dispositivo impreso en 3D para asegurar posicionamiento (Vega et al.). Los cuerpos de prueba fueron radiografiados con equipo X Myray RXAC (Imolia, Italia).

La imagen obtenida con FireCR Dental Reader (3DISC Imaging, Virginia, USA) fue importada al Software PlanmecaRomexis 4.4.0,R (Planmeca, Helsinki, Finland). Posteriormente la imagen fue exportada en formato TIFFy analizada con escala de grises en Adobe Photoshop CS7 (Adobe, California, USA), seleccionando un área representativa tanto de la muestra (200 x 100 pixeles), como de la cuña de aluminio (100 $x 500$ pixeles). El valor de escala de grises varía entre 0 y 255 , donde 0 representa al negro puro y 255 al blanco puro.
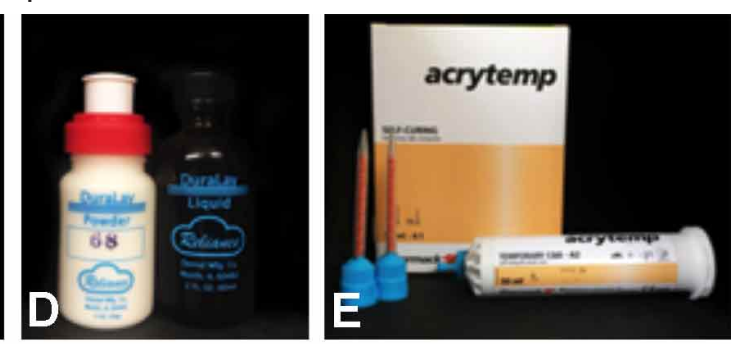

Fig. 1. Resinas acrílicas de polimetilmetacrilato (a)Acrílico Coronas Autocurable, Marché®, Chile (b) Alike ${ }^{\mathrm{TM}}$ Temporary Crown and Bridge Resin, GC America, EEUU (c) Caulk® Temporary Bridge Resin, Dentsply, EEUU (d) DuralayTemporary Crown \& Bridge, Reliance, EEUU, y resinas bisacrílicas, EEUU (e)Acrytemp, Zhermack, Italia (f) Luxacrown, DMG, Alemania (g) Structur Premium, Voco, Alemania (h) LuxatempStar, DMG, Alemania (i)ExperTemp, Ultradent, EEUU (j) Protemp ${ }^{\text {TM }} 4$, 3M ESPE, EEUU. 
Análisis Estadístico. Los datos de radiopacidad equivalente de las muestras de cada grupo, expresada en milímetros de aluminio, se analizaron con el software SPSS Statistics 21,0 (IBM, New York, USA). Se utilizó test de Shapiro-Wilk para determinar normalidad de la distribución de los datos de cada material. Después de probar la normalidad, los datos se analizaron con ANOVA de un factor y HSD Tukey, con nivel de significancia de $p \leq 0,05$.

\section{RESULTADOS}

Radiopacidad de Resinas acrílicas. Se determinó la existencia de diferencias significativas entre los materiales estudiados. La resina acrílica ALIKE ${ }^{\mathrm{TM}} \mathrm{GC}$ presentó una radiopacidad estadísticamente mayor $(0,85$ $\pm 0,08 \mathrm{~mm}$ de Al) que las otras resinas acrílicas estudiadas. La resina Marché presentó una radiopacidad de 0,72 $\pm 0,32 \mathrm{~mm}$ de Al,CaulkâTemporary Bridge Resinde 0,65 $\pm 0,25$ y Duralay de 0,56 $\pm 0,05$ (Fig. 2).

Radiopacidad de Resinas Bisacrílicas. Las resinas bisacrílicasAcrytemp, Luxacrown, LuxatempStar y Structur Premium presentaron una mayor radiopacidad equivalente que las resinas bisacrílicasExpertemp y Protemp ${ }^{\mathrm{TM}}$ (Fig. 3). En orden descendiente la radiopacidad equivalente de las resinas bisacrílicas fue de 1,75 $\pm 0,2$ para Acrytemp, 1,51 $\pm 0,27$ para Luxacrown, 1,47 \pm 0,14 para Structur Premium, 1,43 \pm 0,23 para LuxatempStar, $1,02 \pm 0,28$ para Protemp ${ }^{\text {TM }} 4$ y $1 \pm 0,1$ para ExperTemp ${ }^{\mathrm{TM}}$

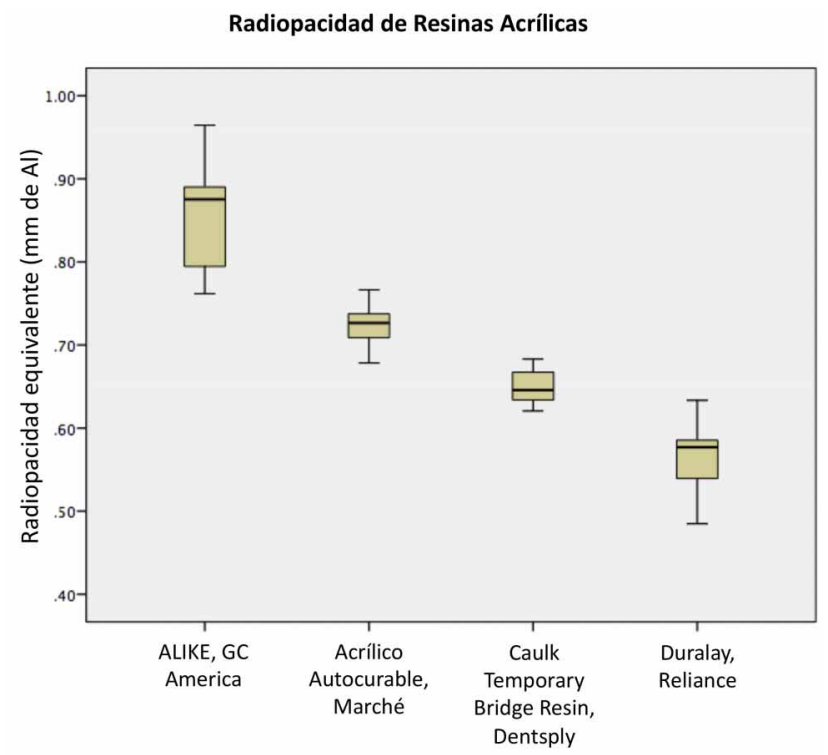

Fig. 2. Radiopacidad equivalente en milímetros de Al de resinas acrílicas evaluadas.

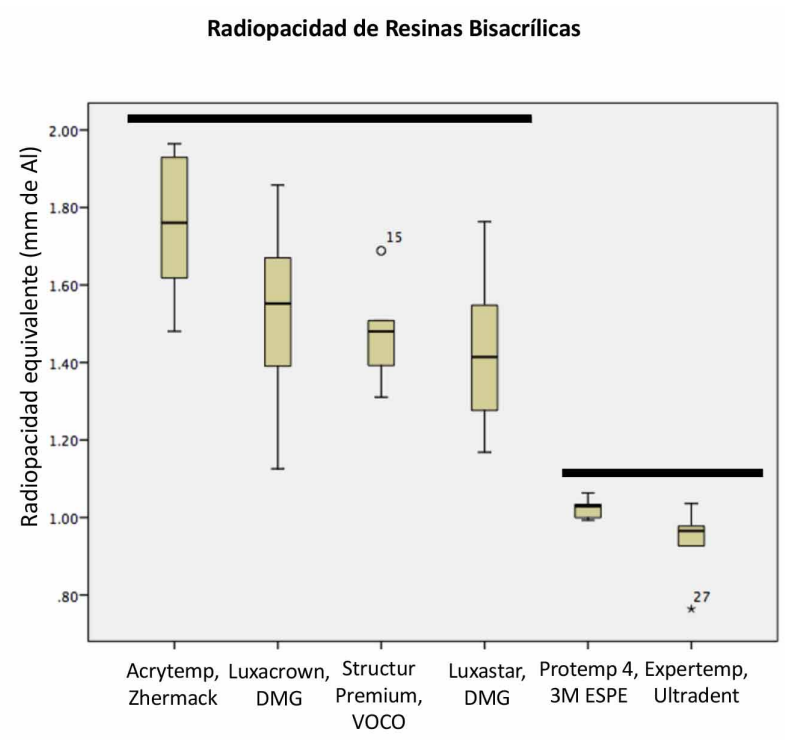

Fig. 3. Radiopacidad equivalente en milímetros de Al de resinas bisacrílicas evaluadas. Barras horizontales indican grupos de materiales con radiopacidades estadísticamente diferentes $(p<0,05)$.

\section{DISCUSIÓN}

El presente estudio buscó evaluar la radiopacidad de dos tipos de materiales para la fabricación de provisionales disponibles; las resinas acrílicas y bisacrílicas. Se demostró que existen diferencias significativas en estos grupos de materiales estudiados. Más aún, de acuerdo al requisito de radiopacidad establecido por la norma ISO 4049: "Materiales restauradores basados en polímeros" (International Organization for Standardization, 2009), se demostró que mientras las resinas bisacrílicas sí cumplen, las resinas acrílicas no cumplen con tener una radiopacidad equivalente de al menos $1 \mathrm{~mm}$ de aluminio.

Es importante la evaluación de la radiopacidad de una restauración provisional, ya que su evaluación con examen radiográfico permite evaluar la efectividad terapéutica de los tratamientos rehabilitadores (Blasi \& Barrero, 2011). Si bien, la clínica es imperante al realizar un correcto diagnóstico, la radiografía es un examen complementario que ayuda a facilitar el diagnóstico sobre todo en zonas de difícil acceso clínico. Mediante el examen radiográfico se pueden descartar o pesquisar la presencia de brechas marginales y sobrecontornos proximales en la restauración provisoria, los que pueden producir inflamación y generar problemas futuros, por ejemplo, en la toma 
de impresión o cementación de la restauración definitiva, y cobran gran importancia en la mantención de la vitalidad del remanente dentario y en la protección de la contaminación de los tratamientos endodónticos en dientes no vitales (Fox \& Gutteridge, 1997). La radiopacidad de las restauraciones provisionales también permite localizar fragmentos de estos en zonas no deseadas, como lechos quirúrgicos en la carga inmediata de implantes, áreas subgingivales en cirugías de ancho biológico, furcas en dientes con compromiso del estado periodontal y fragmentos de material en caso de iatrogenia, impactación y/o aspiración de este tipo de restauraciones, evitando complicaciones que puedan alterar a los tejidos blandos adyacentes (Mattie et al.; Romanos).

La radiopacidad de los materiales dentales está determinada por su composición, siendo los más comunes agentes radiopacos: óxido de bismuto, sulfato o fosfato de calcio, sulfato de bario, dióxido de zirconio, dióxido de silicio y dióxido de titanio (Choi, 2015). Se ha establecido que el tipo, la concentración y el tamaño de la partícula del radiopacificador, afectan el nivel de radiopacidad de los distintos materiales dentales (Duarte et al., 2009). En las resinas bisacrílicas, el agente radiopaco se agrega a la composición de su relleno inorgánico, similar a la que ocurre con las resinas compuestas (Comisi, 2015). En el caso de las resinas acrílicas el agente radiopaco se encuentra en el polímero (polvo), pero el porcentaje que se agrega del agente radiopaco es menor, ya que su aumento va en desmedro de las propiedades físicas y estéticas de este material (Bloodworth \& Render, 1992), lo que explica la diferencia de radiopacidad entre ambos grupos de materiales utilizados para restaurar en forma provisoria.

Las resinas bisacrílicas según diversos estudios presentan mejoras en las distintas propiedades de los materiales para provisionalización, entre ellos una menor contracción de polimerización, menor daño a la pulpa y a los tejidos gingivales, y algunos estudios también reportan que presentan mejor adaptación marginal (Abdulmohsen et al., 2016). De manera similar, el presente estudio demuestra mejores propiedades de radiopacidad de las resinas bisacrílicas en comparación a las resinas acrílicas, lo cual es también relevante, ya que fueron sólo estos materiales, del grupo total de materiales estudiados, los que cumplieron con los requisitos establecidos por la normativa ISO4049, que exige una radiopacidad de al menos 1 milímetro de aluminio.
Dentro de las resinas bisacrílicas estudiadas, Acrytemp (Zhermack) presentó la mayor radiopacidad, 1,75 $\pm 0,25 \mathrm{~mm}$ de Al. El fabricante, Zhermack, no especifica que elemento radiopacificador utiliza (Zhermack, 2016). Los materiales bisacrílicos, LuxatempStar y LuxCrown (DMG, 2017), presentaron radiopacidad de $1,43 \pm 0,23$ y 1,51 $\pm 0,27 \mathrm{~mm}$ de Al respectivamente, cumpliendo también con la norma exigida por ISO 4049. Para ambos materiales el fabricante describe su composición solo como acrilato-resina sin mayores especificaciones en sus hojas de seguridad (DMG). De manera similar, VOCO fabricante de la resina bisacrílicaStructur Premium, tampoco revela mayores detalles de su composición, más allá de que contiene metacrilatos, aminos, terpenos, benzoil-peróxido y BHT (VOCO, 2017). En el presente estudio se determinó una radiopacidad equivalente de 1,47 $\pm 0,14 \mathrm{~mm}$ de aluminio, similar a lo observado en otros reportes (Pekkan, 2011). La resina bisacrílica Protemp ${ }^{\mathrm{TM}} 4$ presentó una radiopacidad de 1,02 $\pm 0,28 \mathrm{~mm}$ de aluminio, de acuerdo a su fabricante este material presenta una nueva generación de relleno sofisticado, sin embargo, no se encontraron mayores detalles de él (3M). Finalmente, dentro de este grupo, el material Expertemp (Ultradent) presentó una radiopacidad equivalente a $1 \pm 0,10 \mathrm{~mm}$ de aluminio, la cual solo se aproxima a la norma 4049 exigida por ISO.

En contraste a los resultados de radiopacidad obtenidos por las resinas bisacrílicas, en el presente estudio se determinó que las resinas acrílicas testeadas no cumplen con el requisito de radiopacidad exigido por la norma ISO 4049: "Materiales restauradores basados en polímeros" (International Organization for Standardization, 2009). Dentro de este grupo, la resina acrílica ALIKE TM GC (GCAmerica), fue la que presentó mayor valor de radiopacidad $(0,85 \pm 0,08 \mathrm{~mm}$ de $\mathrm{Al})$, a pesar de ser la más alta es este grupo de materiales, no alcanza a cumplir con la norma exigida por ISO. Los otros materiales evaluados (Acrílico de Coronas Autocurable Marché, Temporary Bridge ResinCaulk $\AA$, Dentsply y Temporary Crown and Bridge Duralay, Reliance) presentaron valores promedios de radiopacidad menores a lo exigido por la normativa ISO 4049.

Otros autores han reportado la dificultad para visualizar las resinas acrílicas en la imagen radiográfica (Alvarez, 1966; Newton et al., 1987; Bloodworth \& Render, 1992; Mattie et al.). Estudios que evalúan la radiopacidad de las resinas acrílicas son escasos y fueron la mayoría publicados en la década de los no- 
venta. La baja radiopacidad de las resinas acrílicas, se suma a las desventajas que presentan estos materiales, entre ellas, su alta contracción de polimerización, elevada cantidad de residuos monoméricos y alta exotermia. Sin embargo, éstos materiales siguen estando vigentes y son de elección para muchos dentistas debido a su bajo costo y aceptable adaptación marginal, es por este motivo, que se sugiere estudiar y evaluar nuevos componentes químicos que otorguen radiopacidad a estos materiales sin alterar sus propiedades.

Por otro lado, es importante considerar que las restauraciones provisionales pueden permanecer en boca por cantidades considerables de tiempo, sobre todo en tratamientos complejos, que involucren rehabilitaciones de boca completa. En estos casos las restauraciones deber ser evaluadas constantemente en el tiempo, sobre todo porque los cementos para provisionalización presentan una rápida disolución (Gratton \& Aquilino, 2004). En este tipo de rehabilitaciones, las restauraciones provisionales sirven de guía para un correcto diagnóstico, tanto estético como funcional, facilitando la planificación y ejecución de las rehabilitaciones definitivas (Wassell et al., 2002; Strassler, 2013). Por ejemplo, con restauraciones provisorias, se puede realizar análisis del plano oclusal y de la dimensión vertical que tendrán las futuras rehabilitaciones a realizar y si estas son radiopacas, permitirían determinar dichos parámetros a través del análisis cefalométrico en la telerradiografía lateral. Schulz et al. (2013) describen la utilización de láminas de plomo en rodetes de cera para determinar la guía anterior y el plano oclusal en pacientes desdentados parciales a través de la telerradiografía lateral. En estos casos el uso de resinas bisacrílicas, debido a los niveles de radiopacidad que presentan, expanden el campo de uso de los antiguos provisorios, hacia nuevas técnicas, como el mock up, que sirve, entre otros, como guía para permitir la evaluación de la dimensión vertical, la inclinación del plano oclusal y la inclinación de las piezas dentarias en pacientes desdentados parciales o con grandes atriciones dentales tanto en telerradiografías laterales como en la práctica clínica (Vailati \& Belser, 2008). De esa forma, utilizando restauraciones provisorias con resinas bisacrílicas, debido a su adecuada radiopacidad, sería posible analizar radiográficamente distintos parámetros cefalométricos, que ayuden a planificar rehabilitaciones definitivas, sin la necesidad de agregar otros aditamentos.

La radiopacidad de los materiales dentales es una propiedad fundamental, debido a que este exa- men complementario permite realizar diagnósticos más precisos y mejor control de los tratamientos (Pekkan, 2011, 2016). La presente investigación entrega información sobre la radiopacidad de materiales de restauración para provisionalización de incrustaciones, puentes y coronas presentes en el mercado nacional, con la finalidad de ser una referencia tanto para el clínico a la hora de elegir un material, como para el fabricante a la hora de estandarizar y publicitar la propiedad de radiopacidad de sus materiales.

\section{AGRADECIMIENTOS}

Los autores agradecen a VOCO y Claudio Gutiérrez, asesor técnico de Trema Dental; Dental Store y Claudio Arriagada, representantes oficiales de DMG en Chile; ExpressDent y Yamilet Flores, representantes oficiales de GC AMERICA en Chile; Pareja Lecaros representantes oficiales de Zhermack en Chile; 3M ESPE Chile, Paola Culaciati, Juana Espina Alvarado, Asistentes CRM Oral Care 3M Chile; Tecnolmport y Macarena Vargas representantes oficiales de Ultradent, en Chile; Maria Fernanda Snijderm, ClinicalEducator Dentsply Sirona, de Dentsply; Medicaltek S.Ay Alfonso Bacquet de Duralay, Reliance; Marché y al Dr. Javier Martin, por facilitar donar los materiales utilizados y entregar información técnica de los productos.

VEGA, V.; OSORIO, S.; ESTAY, J.; FARFÁN, J.; QUINTANA, C. \& CORRAL, C. Radiopacity of acrylic and bisacrylicresinsused as provisionalrestorations. Int. J. Odontostomat., 15(1):119-124, 2021.

ABSTRACT: An adequate radiopacity of materials for provisionalization allows its proper evaluation on the radiographicexam. However, there is a lack of knowledge about compliance with the requirements established by the ISO 4049 norm. The aim of this study was to determine the radiopacity of provisionalization materials available in the national market and verify if they comply with the minimum requirements of ISO regulations. An in vitro experimental study was carried out, samples of $10 \mathrm{~mm}$ in diameter and 1 $\mathrm{mm}$ thickness of acrylic resins: Self-Cure Acrylic (Marché $®$ ), Duralay (Reliance), Caulkâ (Dentsply), Alike ${ }^{\mathrm{TM}}(\mathrm{GC})$ and by sacrilic resins: Protemp ${ }^{\mathrm{TM}} 4(3 \mathrm{M})$, Luxatemp Star (DMG), Luxacrown (DMG), Structur Premium (VOCO), ExperTemp ${ }^{\text {TM }}$ (Ultradent),Acrytemp (Zhermack) were prepared. The samples were $\mathrm{x}$-rayed together with a stepped aluminum wedge. The images were analyzed to determine the gray scale value and its equivalent value in mm of Aluminum. The data was analyzed to verify compliance with the ISO stan- 
dard. Statistically significant differences were found. The equivalent radiopacity of acrylicresins in $\mathrm{mm}$ of $\mathrm{Al}$ in descending order was $0.85 \pm 0.08$ for ALIKE TM, $0.72 \pm 0.32$ for acrylic Marché $囚, 0.65 \pm 0.25$ forCaulkâ and $0.56 \pm 0.05$ forDuralay, all being less than the required by norm. The equivalent radiopacity of by sacrilic resins was $1.75 \pm 0.2$ for Acrytemp, $1.51 \pm 0.27$ for Luxacrown, $1.47 \pm 0.14$ for Structur Premium, $1.43 \pm 0.23$ for LuxatempStar, $1.02 \pm 0.28$ forProtemp ${ }^{\mathrm{TM}} 4$ and $1 \pm 0.1$ forExperTemp ${ }^{\mathrm{TM}}$, which are greater than the requirements. In conclusion, the bisacrylic resins evaluated in this study comply with the radiopacity standard, however the acrylic resins evaluated do not comply.

KEY WORDS: radiopacity, dental materials, acrylicresin.

\section{REFERENCIAS BIBILIOGRÁFICAS}

3M. ESPE. Protemp ${ }^{\mathrm{TM}} 4$ Temporization Material. Technical Data Sheet. 3M, 2008. Disponible en: https://multimedia.3m.com/ mws/media/5193200/protemp-4-technical-data-sheet.pdf

Abdulmohsen, B.; Parker, S.; Braden, M. \& Patel, M. P. A study to investigate and compare the physicomechanical properties of experimental and commercial temporary crown and bridge materials. Dent. Mater., 32(2):200-10, 2016.

Alvarez, L. C. Radiopacity of acrylic resins. Oral Surg. Oral Med. Oral Pathol., 22(3):318-24, 1966.

Blasi, Á. \& Barrero, C. H. Estudio in vitro para comprobar la estabilidad del color de materiales provisionales usados en prostodoncia. Univ. Odontol., 30(65):17-23, 2011,

Bloodworth, K. E. \& Render, P. J. Dental acrylic resin radiopacity: Literature review and survey of practitioners' attitudes. J. Prosthet. Dent., 67(1):121-3, 1992.

Choi, T. Y. Radiographic assessment of some dental restorative materials. Tesis. Pok Fu Lam, University of Hong Kong, 2015. Disponible en: http://dx.doi.org/10,5353/th_b5573058

Comisi, J. C. Provisional materials: advances lead to extensive options for clinicians. Compend. Contin. Educ. Dent., 36(1):54, 56-9, 2015.

DMG. Safety Datasheets. Hamburgo, DMG, 2017. Disponible en: https://www.dmg-dental.com/en/downloads/safety-datasheets/

Duarte, M. A. H.; El Kadre, G. D. O.; Vivan, R. R.; Tanomaru, J. M. G.; Tanomaru Filho, M. \& de Moraes, I. G. Radiopacity of portland cement associated with different radiopacifying agents. J. Endod., 35(5):737-40, 2009.

Fox, K. \& Gutteridge, D. L. An in vitro study of coronal microleakage in root-canal-treated teeth restored by the post and core technique. Int. Endod. J., 30(6):361-8, 1997.

Gómez Mira, F. \& Ardila Medina, C. M. Contornos y perfil de emergencia: aplicación clínica e importancia en la terapia restauradora. Av. Odontoestomatol., 25(6):331-8, 2009.

Gratton, D. G. \& Aquilino, S. A. Interim restorations. Dent. Clin. North Am., 48(2):487-97, 2004.

International Organization for Standardization (ISO). ISO 4049, Dentistry Polymer-based filling, restorative and luting materials. $3 a$ ed. Ginebra, International Organization for Standardization, 2009.

Mattie, P. A.; Rawls, H. R. \& Cabasso, I. Development of a radiopaque, autopolymerizing dental acrylic resin. J. Prosthodont., 3(4):213-8, 1994.
Newton, J. P.; Abel, R. W.; Lloyd, C. H. \& Yemm, R. The use of computed tomography in the detection of radiolucent denture base material in the chest. J. Oral Rehabil., 14(2):193-202, 1987.

Pekkan, G. Comparative radiometric evaluation of some provisional crown and bridge materials. J. Dent. Fac. Atatürk Univ., 21(1):28-32, 2011,

Pekkan, G. Radiopacity of dental materials: an overview. Avicenna J. Dent. Res., 8(2):e36847, 2016.

Romanos, G. E. Present status of immediate loading of oral implants. J. Oral Implantol., 30(3):189-97, 2004.

Schulz, R. R.; Cerda, P. B.; Moya, M. M. \& Romo, O. F. Occlusal plane and anterior guidance visualization in lateral cephalometric radiographs in partially edentulous patients: technique description through case reports. Int. J. Odontostomat., 7(3):46570, 2013.

Strassler, H. E. Fixed prosthodontics provisional materials: making the right selection. Compend. Contin. Educ. Dent., 34(1):22-4, 2013.

Turgut, M. D.; Attar, N. \& Onen, A. Radiopacity of direct esthetic restorative materials. Oper. Dent., 28(5):508-14, 2003.

Vailati, F. \& Belser, U. C. Full-mouth adhesive rehabilitation of a severely eroded dentition: the three-step technique. Part 2. Eur. J. Esthet. Dent., 3(2):128-46, 2008.

Vega, V.; Negrete, P.; Osorio, S.; Estay, J. \& Corral, C. Radiopacity assessment using $3 D$ printed devices. En: XXVIII Reunión Anual de IADR División Chile. Santiago de Chile, IADR, 2017.

VOCO. Structur Premium. Instructions for use. VOCO, 2017. Disponible en: https://www.voco.dental/es/portaldata/1/resources/ products/instructions-for-use/e1/structur-premium_ifu_e1,pdf

Wassell, R. W.; St. George, G.; Ingledew, R. P. \& Steele, J. G. Crowns and other extra-coronal restorations: provisional restorations. Br. Dent. J., 192(11):619-22, 2002.

Zhermack. Acrytemp Solution for short and long-term temporaries. Badia Polesine, Zhermack, 2016 Disponible en: https:// www.zhermack.com/public/uploads/F121102_1904_Brochure_Acrytemp_EN_low.pdf

Dirección para correspondencia:

Camila Corral Núñez

Departamento de Odontología Restauradora

Facultad de Odontología

Universidad de Chile

Olivos 943

Independencia

Santiago

CHILE

Email: camila.corral@odontologia.uchile.cl 